\title{
A Gamma Analysis Approach to Determine Optimal Parameters for Monte Carlo Simulation of 6MV Varian Clinac iX Photon Beam
}

\author{
Roger Cai Xiang Soh ${ }^{1}$, Lloyd Kuan Rui Tan ${ }^{1}$, Mark Sen Liang Goh ${ }^{1}$, Sitti Yani ${ }^{3}$, Freddy Haryanto ${ }^{3}$, Wen Siang \\ Lew $^{1}$ and James Cheow Lei Lee ${ }^{1,2}$ \\ 1. Division of Physics and Applied Physics, School of Physical and Mathematical Sciences, Nanyang Technological University, Singapore \\ 2. Division of Radiation Oncology, National Cancer Centre Singapore, Singapore \\ 3. Department of Physics, Faculty of Mathematics and Natural Sciences, Institut Teknologi Bandung, Indonesia
}

\begin{abstract}
Monte Carlo (MC) method is the gold standard dose calculation algorithm. Determination of the electron beam parameters for MC simulation is often estimated using trial and error methods. However, this can be tedious and time-consuming. This paper aims to validate MC simulated data using $1 \mathrm{D}$ gamma analysis for $6 \mathrm{MV}$ photon beam to obtain the optimal parameters. BEAMnrc codes were used to generate phase space files for conventional field sizes $10 \times 10 \mathrm{~cm}^{2}, 6 \times 6 \mathrm{~cm}^{2}, 4 \times 4 \mathrm{~cm}^{2}$ and small field sizes $2 \times$ $2 \mathrm{~cm}^{2}, 1 \times 1 \mathrm{~cm}^{2}, 0.5 \times 0.5 \mathrm{~cm}^{2}$. For conventional field sizes, simulations were benchmarked against Golden Beam Data (GBD). Simulations for small fields were benchmarked against measurements obtained using EDGE Detector and PTW Diode SRS detector in a Sun Nuclear 3D scanner. Dose profiles in water were calculated using DOSXYZnrc codes. Initial reference parameters were approximated using average percentage dose differences of different mean electron energy and electron beam radial distribution (Full Width at Half Maximum, FWHM). Subsequently, the optimal parameters were validated by 1D gamma analysis using varying gamma criteria from $\gamma_{0.3 \% / 0.3 \mathrm{~mm}}$ to $\gamma_{2.0 \% / 2.0 \mathrm{~mm}}$ for depth dose and lateral dose profiles. Comparisons were performed along the central region at depth dose $1.6 \mathrm{~cm}$. Optimal parameters were found to be unique for small field sizes. As field size decreases, smaller FWHM were required to match measured data. By using $95 \%$ passing rate, a generic set of optimal electron beam parameters in a MC model for all field sizes could be accurately determined. Our findings provide MC users a set of optimal parameters with sufficient accuracy for MC simulation work.
\end{abstract}

Key words: Monte Carlo, 1D gamma analysis, optimal parameters, small fields.

\section{Introduction}

The MC method has been proven to be the gold standard for dose calculation in radiotherapy [1-3]. However, linac beam models are unique and it is necessary, therefore, to simulate each linac individually to generate a clinically realistic MC simulation [4]. Report of the American Association of Physicists in Medicine (AAPM) Task Group No. 105 highlighted the importance and challenges of using MC method in treatment planning [5].

The first fundamental step is the accurate

Corresponding author: Roger Soh Cai Xiang, Ph.D., research fields: physical and mathematical sciences. determination of the primary electron beam parameters. However, the actual shape and spectra of the electron beam are rarely known. To determine it experimentally requires a portable spectrometer with a degree of sophistication, which is not readily available to radiotherapy clinics $[6,7]$. As a first approximation, the electron beam is modelled as a source with mono-energetic spectra and gaussian spatial distribution by the mean electron energy $(\varepsilon)$ and FWHM. Despite the irregularity of the actual shape of the primary electron beam, a Gaussian model FWHM provides a good initial approximation [6]. Several studies used different procedures for the determination of the electron parameters, such as trial and error 
methods, which can prove to be a difficult task for users as it is tedious and time-consuming [4, 8-10].

Commissioning procedure for a MC model is usually conducted by comparing a set of measurements at a single reference field size (generally $10 \times 10 \mathrm{~cm}^{2}$ ) to the corresponding $\mathrm{MC}$ simulation $[6,11]$. Several studies have reported several different procedures for analysing the accuracy of the simulated profiles $[4,6,8,9,12,13]$.

The objective of this study is to provide an evaluation approach that will clearly identify the optimal parameters for MC simulation. The current study uses 1-dimensional (1D) gamma $(\gamma)$ analysis to evaluate the match of the 6MV photon beam simulated depth doses and lateral profiles of conventional field sizes $\left(10 \times 10 \mathrm{~cm}^{2}, 6 \times 6 \mathrm{~cm}^{2}, 4 \times 4\right.$ $\left.\mathrm{cm}^{2}\right)$ to GBD and small field sizes $\left(2 \times 2 \mathrm{~cm}^{2}, 1 \times 1\right.$ $\mathrm{cm}^{2}, 0.5 \times 0.5 \mathrm{~cm}^{2}$ ) to measured data. Study was done to investigate the optimal parameters differences between conventional and small field sizes. A generic set of optimal electron beam parameters for all field sizes will be also determined.

\section{Experimental Methods}

Small field measurements were obtained by crosschecking measured data from EDGE Detector (Sun Nuclear Corporation, Melbourne, FL) and PTW Diode SRS (PTW-60018) in Sun Nuclear 3D water scanner. To avoid confusion, the term 'measured data' will refer to GBD for conventional field sizes and measured data in small fields.

\subsection{Monte Carlo Simulation Parameters}

Phase space file (phsp) for field size $10 \times 10 \mathrm{~cm}^{2}$ $(100 \mathrm{~cm}$ source to surface distance (SSD)) was generated using BEAMnrc user codes (National Research Council of Canada, Ottawa, ON). Specifications provided from the Varian Monte Carlo data package for $6 \mathrm{MV}$ photon beam from Varian Clinac iX (Varian Medical Systems Inc., Palo Alto, CA) was modeled [14]. Gaussian radial intensity distribution was assigned to the incident electron beam.

DOSXYZnrc user codes calculated the dose distribution in the region of interest. H20700ICRU was used to simulate the water medium. Voxel size was $0.1 \times 0.1 \times 0.1 \mathrm{~cm}^{3}$, which was consistent with the calculation grid size in clinical Eclipse Treatment Planning Systems (TPS). Electron and photon transport thresholds for BEAMnrc and DOSXYZnrc simulations were $0.700 \mathrm{MeV}$ and $0.01 \mathrm{MeV}$ respectively.

\subsection{Simulation of Parameters}

For each of the field sizes, depth dose calculation of 5 different incident mean electron energies $(\varepsilon=$ 5.8-6.2 MeV) were done. Normalisation point was done at $1.6 \mathrm{~cm}$ depth. Comparisons of varying FWHM parameter $(\mathrm{FWHM}=0.1-0.5 \mathrm{~cm})$ were done for the lateral dose profile along the x-axis at depth $1.6 \mathrm{~cm}$.

\subsection{Obtaining Initial Reference Parameters}

Initial reference electron beam parameters and initial reference jaw thickness were obtained by interpolating from the relationship of several $\varepsilon$ and FWHM at reference field size $10 \times 10 \mathrm{~cm}^{2}[11,13]$. These initial reference parameters were obtained by evaluating the best-fit curve of the average percentage dose difference ( $\%$ Difference) between all normalised simulated and measured data, defined as follows:

$$
\% \text { Difference }=\frac{\sum_{\mathrm{n}} \frac{\mathrm{D}_{\mathrm{MC}}-\mathrm{D}_{\text {measureddata }}}{\mathrm{D}_{\text {measureddata }}} \%}{\mathrm{n}}
$$

where $D_{M C}$ and $D_{\text {measureddata }}$ represent Monte Carlo simulated data and measured data respectively; $n$ is the total number of simulated points $[13,15]$.

It was found that $\varepsilon=6.1 \mathrm{MeV}$ and $\mathrm{FWHM}=0.3 \mathrm{~cm}$ initial electron beam was optimal for reference field size $10 \times 10 \mathrm{~cm}^{2}$ based on the average dose difference relationship from Equation 1 (Fig. 1). Variations of other parameters for different field sizes will be referenced to $\varepsilon=6.1 \mathrm{MeV}$ and $\mathrm{FWHM}=0.3 \mathrm{~cm}$. Each time, only one parameter was varied while the other parameter was kept constant for comparison. 


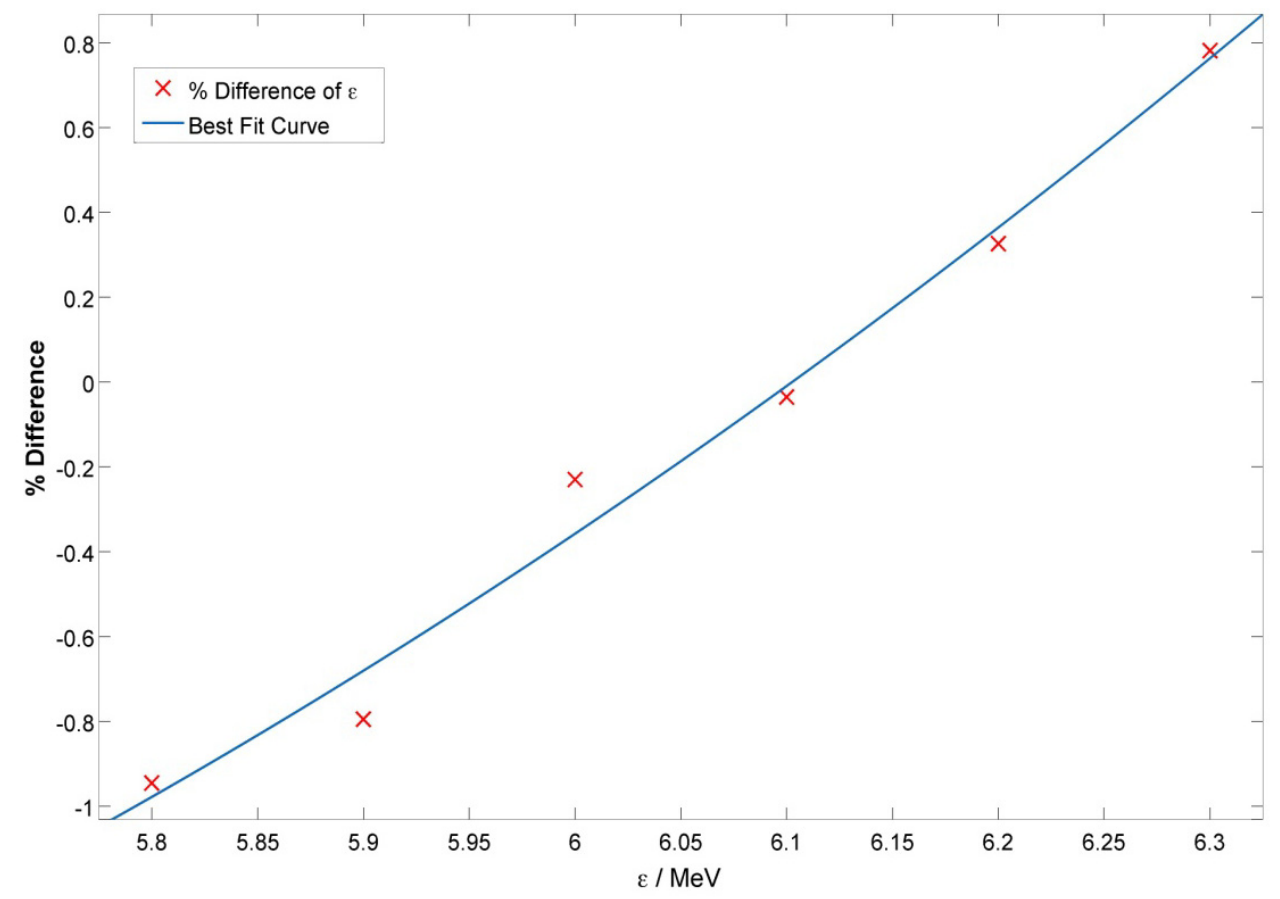

Fig. 1 Relationship between $\varepsilon$ and average dose difference against measured data. The best-fit curve was fitted with a second order polynomial function, with $R^{2}=0.985$. 6.1 MeV was chosen as the initial reference electron energy with the lowest percentage dose difference.

\subsection{D Gamma Analysis}

MC simulated lateral and depth dose profiles were analysed using 1D $\gamma$ analysis as presented by Low et al. [16]. In order to clearly identify the optimal parameters, $\gamma$ criteria were varied between $0.3 \%$ to $2.0 \%$ dose difference and $0.3 \mathrm{~mm}$ to $2.0 \mathrm{~mm}$ distance-to-agreement (DTA) $\quad\left(\gamma_{0.3 / 0.3}-\gamma_{2.0 / 2.0}\right) . \quad$ MC simulated data points with be considered a pass if $\gamma \leq$ 1.0 and fail if $\gamma>1.0$. Determination of the optimal parameters for each field size was based on the highest percentage of passes using the most stringent $\gamma$ criteria, $\gamma_{0.3 / 0.3}$. Parameter with $95 \%$ of the total simulated points that passes using the $\gamma_{0.5 / 0.5}$ criteria (95\% passing rate) were also identified so as to determine a generic set of optimal parameters for all field sizes.

\section{Results}

\subsection{Depth dose Profile Comparison with Varying $\varepsilon$}

In Fig. 2, a clear trend was observed by applying $\gamma$ analysis. This allows the determination of optimal $\varepsilon$ for each field size. For all field sizes, $\gamma_{2.0 / 2.0}$ and $\gamma_{1.0 / 1.0}$ were unable to differentiate the optimal $\varepsilon$ as most of the data points passes the acceptance criteria with $95 \%$ passes. Results show that optimal mean electron energy for field sizes larger than $2 \times 2 \mathrm{~cm}^{2}$ was 6.1 $\mathrm{MeV}$. For field sizes smaller than $2 \times 2 \mathrm{~cm}^{2}$, all of the variations, except $\varepsilon=5.8 \mathrm{MeV}$, passes the $95 \%$ passing rate for the $\varepsilon$ parameter (Figs. $2 \mathrm{a}$ and $2 \mathrm{~b}$ ).

\subsection{Lateral Profile Comparison with Varying FWHM}

In Fig. 3, results show that optimal FWHM parameter decreases with smaller field sizes. At extreme small field size $0.5 \times 0.5 \mathrm{~cm}^{2}$, electron beam distribution was simulated closer to a point source with optimal FWHM $=0.1$. FWHM $=0.3$ was optimal for field sizes greater than $4 \times 4 \mathrm{~cm}^{2}$. Percentage of passes for $\mathrm{FWHM}=0.3$ were within $95 \%$ passing rate for field size $0.5 \times 0.5 \mathrm{~cm}^{2}$ and $1 \times 1 \mathrm{~cm}^{2}$ for $\gamma_{0.4 / 0.4}$.

\subsection{Summary of Results}




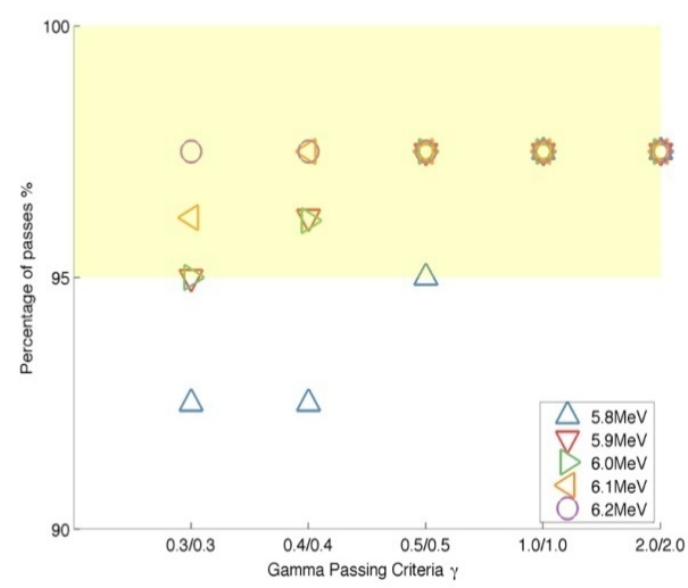

(a)Small field size $0.5 \times 0.5 \mathrm{~cm}^{2}$

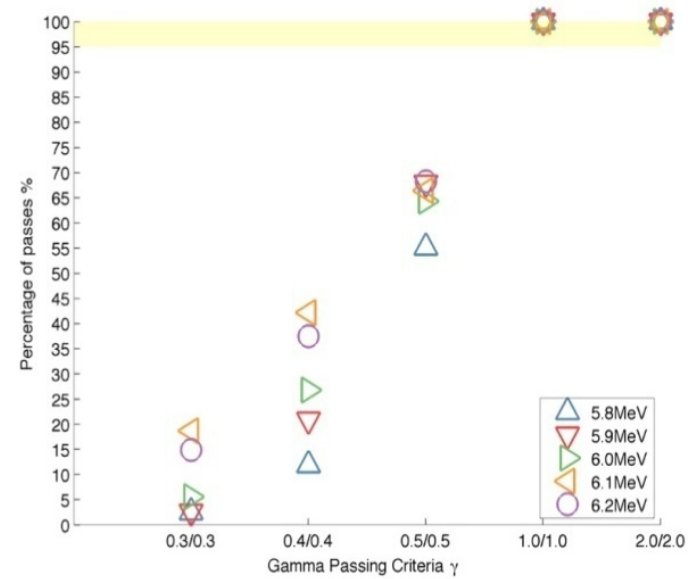

(c)Small field size $2 \times 2 \mathrm{~cm}^{2}$

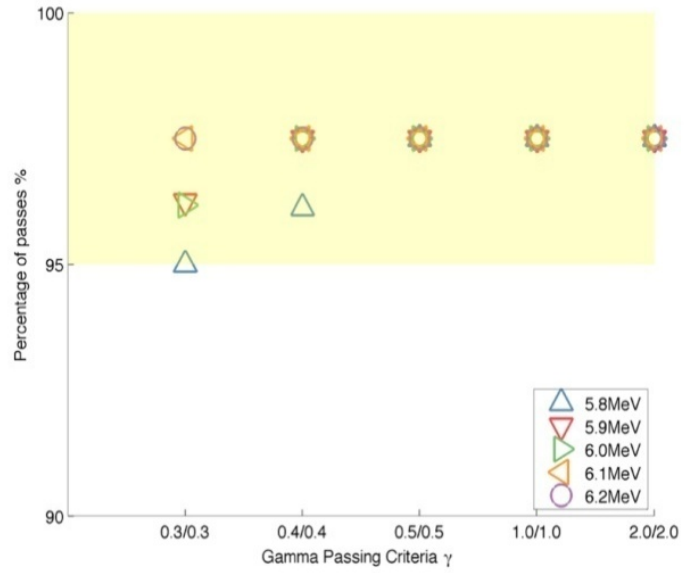

(b)Small field size $1 \times 1 \mathrm{~cm}^{2}$

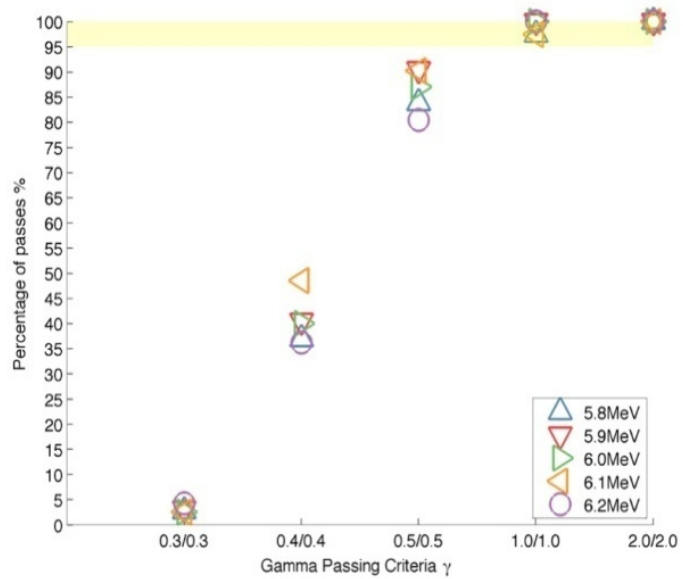

(d)Conventional field size $4 \times 4 \mathrm{~cm}^{2}$

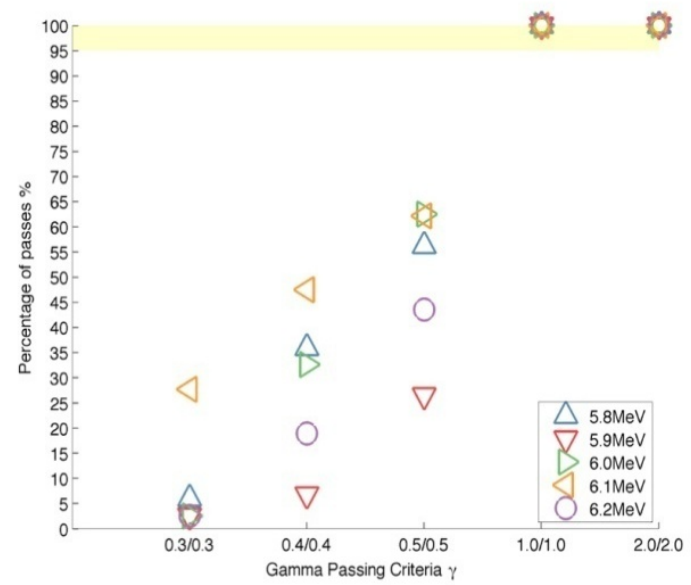

(f)Conventional field size $10 \times 10 \mathrm{~cm}^{2}$

(e)Conventional field size $6 \times 6 \mathrm{~cm}^{2}$

Fig. $2 \gamma$ analysis of the depth dose profile with varying $\varepsilon$ for small and conventional field sizes. 


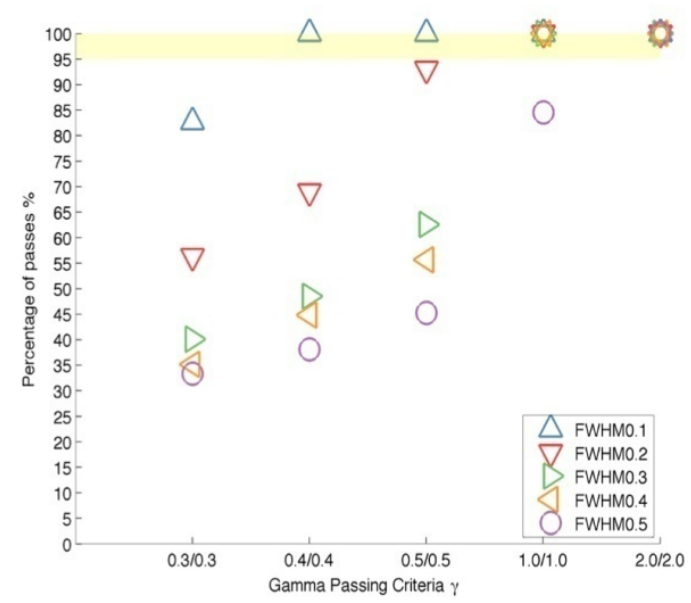

(a)Small field size $0.5 \times 0.5 \mathrm{~cm}^{2}$

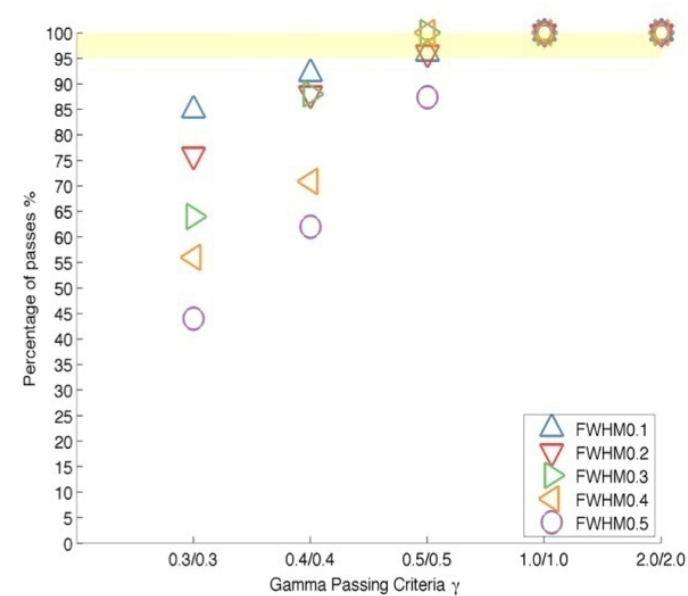

(c)Small field size $2 \times 2 \mathrm{~cm}^{2}$

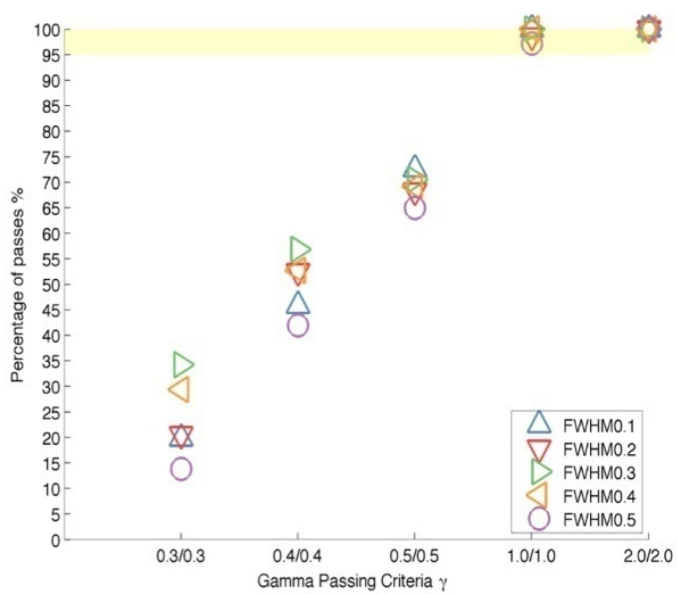

(e)Conventional field size $6 \times 6 \mathrm{~cm}^{2}$

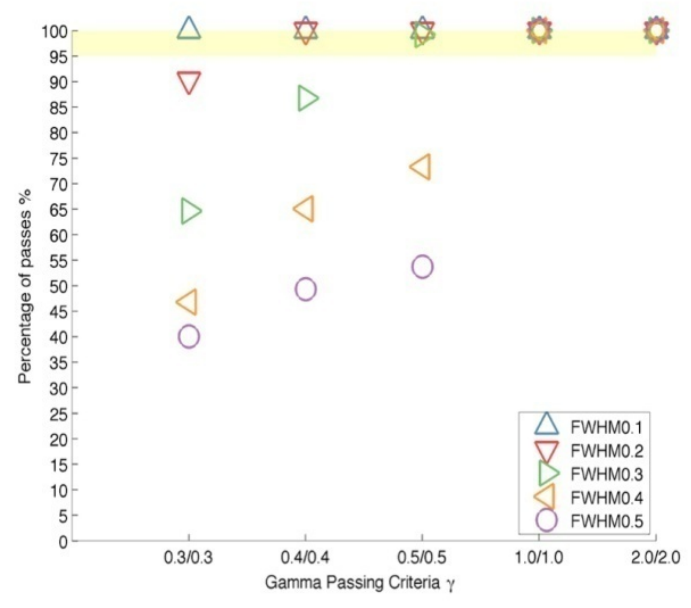

(b)Small field size $1 \times 1 \mathrm{~cm}^{2}$

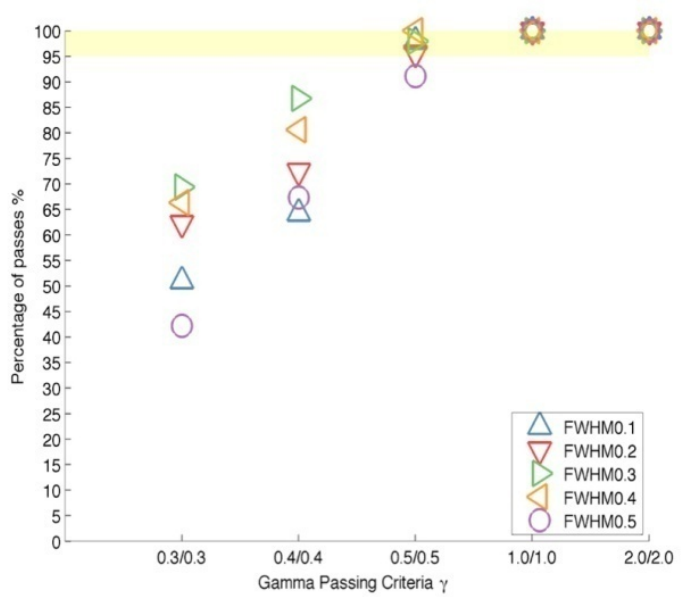

(d)Conventional field size $4 \times 4 \mathrm{~cm}^{2}$

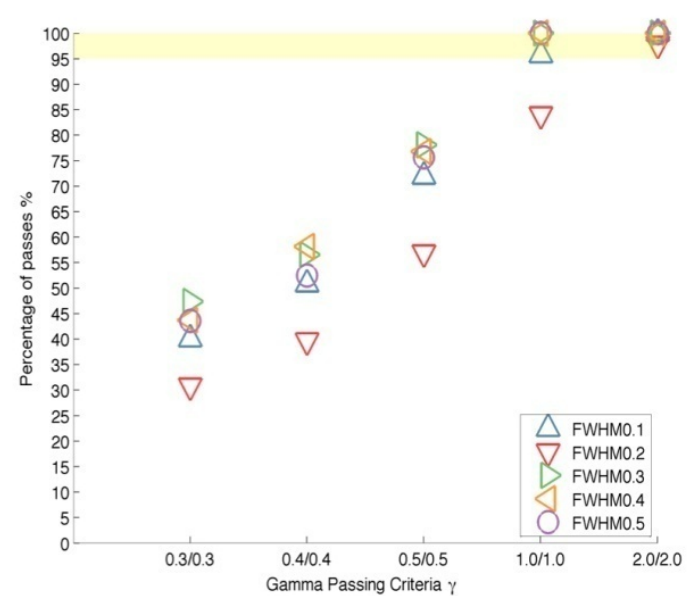

(f)Conventional field size $10 \times 10 \mathrm{~cm}^{2}$

Fig. $3 \gamma$ analysis of the lateral dose profile with varying FWHM for small and conventional field sizes. 
Table 1 Optimal Parameters for MC simulation of measured data based on 1D $\gamma$ analysis.

\begin{tabular}{lll}
\hline Field Size & Mean Electron Energy/ $\varepsilon(\mathrm{MeV})$ & FWHM $(\mathrm{cm})$ \\
\hline FS0.5 & $5.8-6.1$ & 0.1 \\
FS1 & $5.9-6.2$ & 0.1 \\
FS2 & 6.1 & 0.1 \\
FS4 & 6.1 & 0.3 \\
FS6 & 6.1 & 0.3 \\
FS10 & 6.1 & 0.3 \\
\hline
\end{tabular}

Table 2 Optimal Parameters for all field sizes based on $95 \%$ passes with $\gamma_{0.5 / 0.5}$ criteria.

\begin{tabular}{ll}
\hline Mean Electron Energy/ $\varepsilon(\mathrm{MeV})$ & FWHM $(\mathrm{cm})$ \\
\hline 6.1 & 0.3 \\
\hline
\end{tabular}

\section{Discussion}

Optimal parameters for $\mathrm{MC}$ simulated data were obtained using 1D gamma analysis with varying gamma criteria from $\gamma_{0.3 \% / 0.3 \mathrm{~mm}}$ to $\gamma_{2.0 \% / 2.0 \mathrm{~mm}}$ for depth dose and lateral dose profiles.

It was found that variations in $\varepsilon$ have greater dependence on the depth dose profile as compared to FWHM variations. Results were consistent to study done by Chetty et al. [5], where it was reported that incident electron radial distribution have little observable effect on central-axis depth dose.

Similarly, variations in $\varepsilon$ have weak dependence on the lateral dose profile as compared to FWHM variations. Most of the data points due to the variations in $\varepsilon$ passes the acceptance criteria with $95 \%$ confidence. It was found from previous studies that the FWHM parameter affects the central and penumbra regions, particularly the 'horns' of the lateral profile [5-7].

Initially, dose profiles were compared with tolerance of $2 \%$ difference between measured and simulated doses $[17,18]$. However, it was found that in high-dose gradient regions such as penumbra and the out-of-field regions, a small spatial error results in great percentage differences between measured data and simulation $[16,19,20]$. Therefore, it is difficult to compare the trend using a simple dose- difference distribution across different field sizes. The concept of a distance-to-agreement (DTA) distribution by using $\gamma$ analysis allows users to determine the reliability of the dose calculation. It allows a good measure for regions of high-dose gradients such as penumbra and out-of-field doses, and was critical in regions of a low-dose gradients, where small dose differences results in a high $\gamma$ index [20]. Van Dyk et al. [21] provided guidelines and recommended $\gamma$ criterion to be $\gamma_{3 \% / 4 \mathrm{~mm}}$ for the evaluation of TPS algorithms for photon beams. As MC simulation will often be used as a gold standard dose calculation benchmark, the extremely stringent $\gamma$ passing criteria used in this study will be robust to determine the optimal parameters for a good simulation model.

Previous studies suggested that the determination of the optimal parameters was obtained using a set of measurements of a single field size, generally $10 \times 10$ $\mathrm{cm}^{2}$ or $20 \times 20 \mathrm{~cm}^{2}$ to their correspondent simulations $[12,13,22]$. This method of determination will be acceptable provided that the geometrical configuration of the linac and the primary radiation source are well defined [9]. Results show that an independent determination of optimal parameters, especially for small field sizes, will be required for high accuracy [6]. In this study, it was found that each field size has its unique set of optimal parameters and should be tuned individually to pass a stringent $\gamma$ criteria of $\gamma_{0.3 / 0.3}$, especially for field sizes smaller than $2 \times 2 \mathrm{~cm}^{2}$ [22].

However, imposing a stringent criteria and simulating unique parameters for different field sizes may not be practical, as users may wish to use one set 
of parameters for all field sizes for $\mathrm{MC}$ simulation work. Based on this study, optimal set of parameters for all field sizes can be derived by choosing parameters that have at least a $95 \%$ passing rate with $\gamma_{0.5 / 0.5}$ criteria (Table 2).

\section{Conclusions}

Optimal initial electron beam parameters for small and conventional field sizes were determined using 1D $\gamma$ analysis. Optimal incident mean electron energy and FWHM was unique for field size $2 \times 2 \mathrm{~cm}^{2}$ and below. A set of optimal parameters for all field sizes was determined based on $95 \%$ passing rate with $\gamma_{0.5 / 0.5}$ criteria. Our findings provide MC users a set of optimal parameters with sufficient accuracy for MC simulation work.

\section{Acknowledgment}

This work was supported by the Singapore National Research Foundation, Prime Minister's Office, under a Competitive Research Programme (Non-volatile Magnetic Logic and Memory Integrated Circuit Devices, NRF-CRP9-2011-01), and an Industry-IHL Partnership Program (NRF2015-IIP001-001). The work was also supported by a MOE-AcRF Tier 2 Grant (MOE 2013-T2-2-017). W S Lew is a member of the Singapore Spintronics Consortium (SG-SPIN).

\section{References}

[1] Gagne I. M., and Zavgorodni, S. 2007. "Evaluation of the analytical anisotropic algorithm (AAA) in an extreme water-lung interface phantom using Monte Carlo dose calculations." Journal of Applied Clinical Medical Physics 8 (1).

[2] Ojala, J. 2014. "The accuracy of the Acuros XB algorithm in external beam radiotherapy-a comprehensive review." International Journal of Cancer Therapy and Oncology 2 (4).

[3] Knöös, T., Wieslander, E., Cozzi, L. et al. 2006. "Comparison of dose calculation algorithms for treatment planning in external photon beam therapy for clinical situations." Physics in Medicine and Biology 51 (22): 5785.

[4] Jiang, S. B., Kapur, A., and Ma, C.-M. 2000. "Electron beam modeling and commissioning for Monte Carlo treatment planning.” Medical Physics 27 (1): 180-91.

[5] Chetty, I. J., Curran, B., Cygler, J. E. et al. 2007. "Report of the AAPM Task Group No. 105: Issues associated with clinical implementation of Monte Carlo-based photon and electron external beam treatment planning." Medical Physics 34 (12): 4818-53.

[6] Pena, J., Gonzalez-Castano, D. M., Gomez, F. et al. 2007. "Automatic determination of primary electron beam parameters in Monte Carlo simulation." Med. Phys. 34 (3): 1076-84.

[7] Nahum, A. 2007. Handbook of Radiotherapy Physics. Taylor \& Francis, USA.

[8] Bush, K., Zavgorodni, S., and Beckham, W. 2009. "Inference of the optimal pretarget electron beam parameters in a Monte Carlo virtual linac model through simulated annealing." Medical Physics 36 (6): 2309-19.

[9] Sheikh-Bagheri, D., and Rogers, D. W. O. 2002. "Sensitivity of megavoltage photon beam Monte Carlo simulations to electron beam and other parameters." Medical Physics 29 (3) : 379-90.

[10] Juste, B., Miró, R., Verdú, G., et al. 2012. "Uncertainty and sensitivity analysis of the effect of the mean energy and FWHM of the initial electron fluence on the Bremsstrahlung photon spectra of linear accelerators." Applied Radiation and Isotopes 70 (7): 1267-71.

[11] Pena, J., Franco, L., Gómez, F., et al. 2004. "Commissioning of a medical accelerator photon beam Monte Carlo simulation using wide-field profiles." Physics in Medicine and Biology 49 (21): 4929.

[12] Tzedakis, A., Damilakis, J. E., Mazonakis, M., et al. 2004 "Influence of initial electron beam parameters on Monte Carlo calculated absorbed dose distributions for radiotherapy photon beams." Medical Physics 31 (4): 907-13.

[13] Chang, K.-P., Wang, Z.-W., Shiau, A.-C. 2014. "Determining optimization of the initial parameters in Monte Carlo simulation for linear accelerator radiotherapy." Radiation Physics and Chemistry 95: 161-5.

[14] Varian Medical Systems, Inc. 2008. Monte Carlo Data Package: High energy Accelerator. Report 100040466-02.

[15] Kry, S. F., Titt, U., Pönisch F., et al. 2006. "A Monte Carlo model for calculating out-of-field dose from a Varian 6MV beam." Medical Physics 33 (11): 4405-13.

[16] Low, D. A., Harms, W. B., Mutic, S., et al. 1998. "A technique for the quantitative evaluation of dose distributions." Medical Physics 25 (5): 656-61.

[17] Venselaar, J., and Welleweerd, H. 2001. "Application of a test package in an intercomparison of the photon dose calculation performance of treatment planning systems used in a clinical setting." Radiotherapy and Oncology 60 
(2): 203-13.

[18] Venselaar, J., Welleweerd, H., and Mijnheer, B. 2001. "Tolerances for the accuracy of photon beam dose calculations of treatment planning systems." Radiotherapy and Oncology 60 (2): 191-201.

[19] Hogstrom, K. R., Mills, M. D., Eyer, J. A., et al. 1984. "Dosimetric evaluation of a pencil-beam algorithm for electrons employing a two-dimensional heterogeneity correction." International Journal of Radiation Oncology Biology Physics 10 (4): 561-9.

[20] Harms, W. B., Low, D. A., Wong, J. W., et al. 1998. “A software tool for the quantitative evaluation of 3D dose calculation algorithms." Medical Physics 25 (10): 1830-6.

[21] Dyk, J. V., Barnett, R. B., Cygler, J. E., et al. 1993. "Commissioning and quality assurance of treatment planning computers." International Journal of Radiation Oncology Biology Physics 26 (2): 261-73.

[22] Hartmann Siantar, C. L., Walling, R. S., Daly, T. P., et al. 2001. "Description and dosimetric verification of the PEREGRINE Monte Carlo dose calculation system for photon beams incident on a water phantom." Medical Physics 28 (7): 1322-37. 